\title{
REVIEW
}

\section{Rituximab in the Treatment of Pemphigus Vulgaris}

\author{
Labib R. Zakka · Shawn S. Shetty • A. Razzaque Ahmed
}

To view enhanced content go to www.dermtherapy-open.com

Received: September 17, 2012 / Published online: November 15, 2012

(c) The Author(s) 2012. This article is published with open access at Springerlink.com

\section{ABSTRACT}

Introduction: Rituximab is increasingly used in patients with pemphigus vulgaris (PV) who are nonresponders to conventional therapy.

Methods: A PubMed search was conducted using the words pemphigus vulgaris and rituximab therapy from papers published between 2000 and 2012. Two protocols were used. In the lymphoma protocol, patients received four weekly infusions of rituximab (dose $375 \mathrm{mg} / \mathrm{m}^{2}$ ). The rheumatoid arthritis (RA) protocol consisted of two infusions of $1,000 \mathrm{mg}$ each 15 days apart. The variables recorded from each study included clinical remission off or on therapy, relapse rate, incidence of serious adverse events, concomitant therapies, duration of follow-up,

L. R. Zakka · S. S. Shetty · A. R. Ahmed $(\bowtie)$

Center for Blistering Diseases, 697 Cambridge Street 302, Boston, MA 02135, USA

e-mail: arahmedmd@msn.com

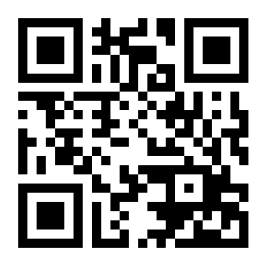

Enhanced content for this article is available on the journal web site: www.dermtherapy-open.com and when available, levels of $\mathrm{B}$ cells and autoantibodies.

Results: Forty-two studies were found, which reported 272 patients; 180 were treated by the lymphoma protocol and 92 by the RA protocol. Both protocols were effective in treating recalcitrant PV. The lymphoma protocol had a lower response rate, relapse rate and serious infections, but higher mortality, and there were nonresponders. The RA protocol produced a higher response rate, relapse rate, number of infections, but lower mortality rate, and lacked nonresponders. The cumulative follow-up for patients treated with the lymphoma protocol was 15.44 months (range 1-41) and 21.04 months (range 8.35-29) for the RA protocol. A major concern in both protocols was the high infection rates, some of which were fatal. A different protocol using a combination of rituximab with intravenous immunoglobulin in a defined manner with a definitive endpoint, used in a limited cohort of patients, showed promising results.

Conclusion: Neither protocol produced a sustained clinical remission and both required continued systemic therapy. Before initiation of treatment, physicians should have a specific 
goal and endpoint and be aware of its potential side effects and lack of information on its longterm effects. Patients should be carefully monitored during and after therapy.

Keywords: Clinical outcomes; Immunology and inflammatory skin diseases; Lymphoma protocol; Pemphigus vulgaris; Rheumatoid arthritis protocol; Rituximab

\section{INTRODUCTION}

Pemphigus vulgaris (PV) is a potentially fatal autoimmune mucocutaneous blistering disease that involves the skin and the mucous membranes [1]. PV is a rare disease with an incidence of approximately $0.1-3.2$ cases per 100,000 individuals annually worldwide [2]. It is a disease of the middle-aged population, typically occurring after the age of 50 years, although some cases have been reported in younger adults and children [3]. PV is seen more frequently in people of Mediterranean decent and Ashkenazi Jews [4]. The incidence in men and women is equal [5].

The histology of PV is an intra-epidermal vesicle with acantholysis [6]. The described antigens are desmoglein 1 (Dsg 1) and desmoglein 3 (Dsg 3) [7]. The immunopathology demonstrates deposition of autoantibodies on keratinocyte cell surfaces and their presence in patients' sera [8].

The mainstay of treatment of PV is systemic corticosteroids. Immunosuppressive agents (ISAs) are used for their steroid-sparing effect and possible ability to reduce autoantibody production [9-11]. Many patients do not respond to high dose long-term corticosteroids in combination with multiple ISAs. Newer methods of treatment, such as rituximab, have shown promise in such patients.
Rituximab is a chimeric monoclonal antibody that targets the CD20 molecule on B cells resulting in their lysis [12]. Pro-B cells, plasmablasts, and plasma cells do not express the CD20 molecule, and are unaffected by rituximab [12]. In 1997, the US Food and Drug Administration approved its use in lymphoma, in 2006 for rheumatoid arthritis (RA), in 2010 for chronic lymphocytic leukemia, and in 2011 for Wegener's granulomatosis [13]. Its use in PV is off label [14]. The rationale for the use of rituximab in patients with $\mathrm{PV}$ is based on its ability to deplete $\mathrm{CD} 20+\mathrm{B}$ cells that presumably produce pathogenic antibodies [12].

The purpose of this review is to provide a critical analysis of the use of rituximab in the treatment of patients with PV.

\section{METHODS}

A PubMed search was conducted using the following keywords: pemphigus vulgaris, rituximab, anti-CD20 monoclonal antibody.

The patients included in this review were derived from studies published between 2000 and the present.

The following inclusion criteria were used: (1) English language; (2) clinical profile consistent with PV; (3) routine histology demonstrating suprabasilar cleft with acantholysis; demonstration of intra-epidermal deposition of immunoreactants on perilesional skin processed by direct immunofluorescence; (5) whenever possible, information on treatments used concomitantly as well as after rituximab therapy; (6) information on dose and frequency of rituximab therapy; (7) provision of clinical outcomes at the end of the study period; (8) occurrence of relapses if they occur, and management of the relapse; (9) reporting the 
length of follow-up; (10) documentation of serious adverse events, especially infections and mortality or lack thereof.

The information retrieved was categorized as follows: patient number, dose of rituximab and number of cycles, concomitant therapies, follow-up duration, adverse effects, clinical outcomes, relapses with re-treatments, levels of B cells, and autoantibody levels. The data are divided into case reports and case series. Case series included a minimum of six patients.

The patients were treated according to the lymphoma or RA protocol with rituximab. The lymphoma protocol consists of four weekly infusions of $375 \mathrm{mg} / \mathrm{m}^{2}$ [14]. The RA protocol consists of two infusions of $1,000 \mathrm{mg} 2$ weeks apart [14].

Clinical outcomes of rituximab therapy included were used as described by Murrell et al. [15]. Complete remission on or off therapy was recorded as reported. In this analysis, partial responders were those patients in whom, after the initiation of rituximab therapy, the dose of systemic corticosteroids and immunosuppressive agents could be reduced by less than $50 \%$ compared to the prerituximab dose. Furthermore, in those patients, clinical disease occurred at intermittent periods, but did not require additional systemic therapy. Nonresponders were those patients who showed no clinical improvement and were considered treatment failures.

\section{RESULTS}

In 42 different publications, information on a total of 272 individual patients with PV treated with rituximab between 2000 and 2012 was available [16-57]. These data were divided into patients treated by (1) the lymphoma protocol, (2) the RA protocol, and (3) modifications or different combinations of either protocol. The information in each of the protocols was divided into case reports and case series.

In the lymphoma protocol, 22 case reports described 48 patients [16-37] and seven case series described 88 patients [43-49]. There are thus 136 patients who were treated by the lymphoma protocol.

There were no case reports in the RA protocol. Four case series described 75 patients [50-53].

Varying and modified versions of the RA or the lymphoma protocols were used within the same group of patients. Ten patients in five case reports got modified versions of the lymphoma protocol [38-42]. There were 51 patients in four different case series [54-57]. Therefore, when the case series and case reports are grouped together, 61 patients received the modified protocols.

The data on these different categories have been summarized in Table 1.

\section{The Lymphoma Protocol}

\section{Case Reports}

The clinical outcomes were as follows [16-37]: complete remission was observed in 32 (66.67\%) patients; nine (18.75\%) off therapy; $21(43.75 \%)$ on therapy; and two $(4.17 \%)$ with unclear treatment status. Nine (18.75\%) were partial responders. Seven (14.58\%) were nonresponders, one of whom after a second cycle had a complete response. The mean duration of follow-up was 12.91 months (range 1-36 months).

Concomitant therapies included: 10 (20.83\%) patients on systemic corticosteroids alone [18, 20, 23, 30, 35, 36]; 36 (75\%) patients on corticosteroids and ISAs [16, 17, 19, 21, 22, 24-26, 28, 29, 31-34, 37]. Two (4.17\%) patients received rituximab as monotherapy [27]. 


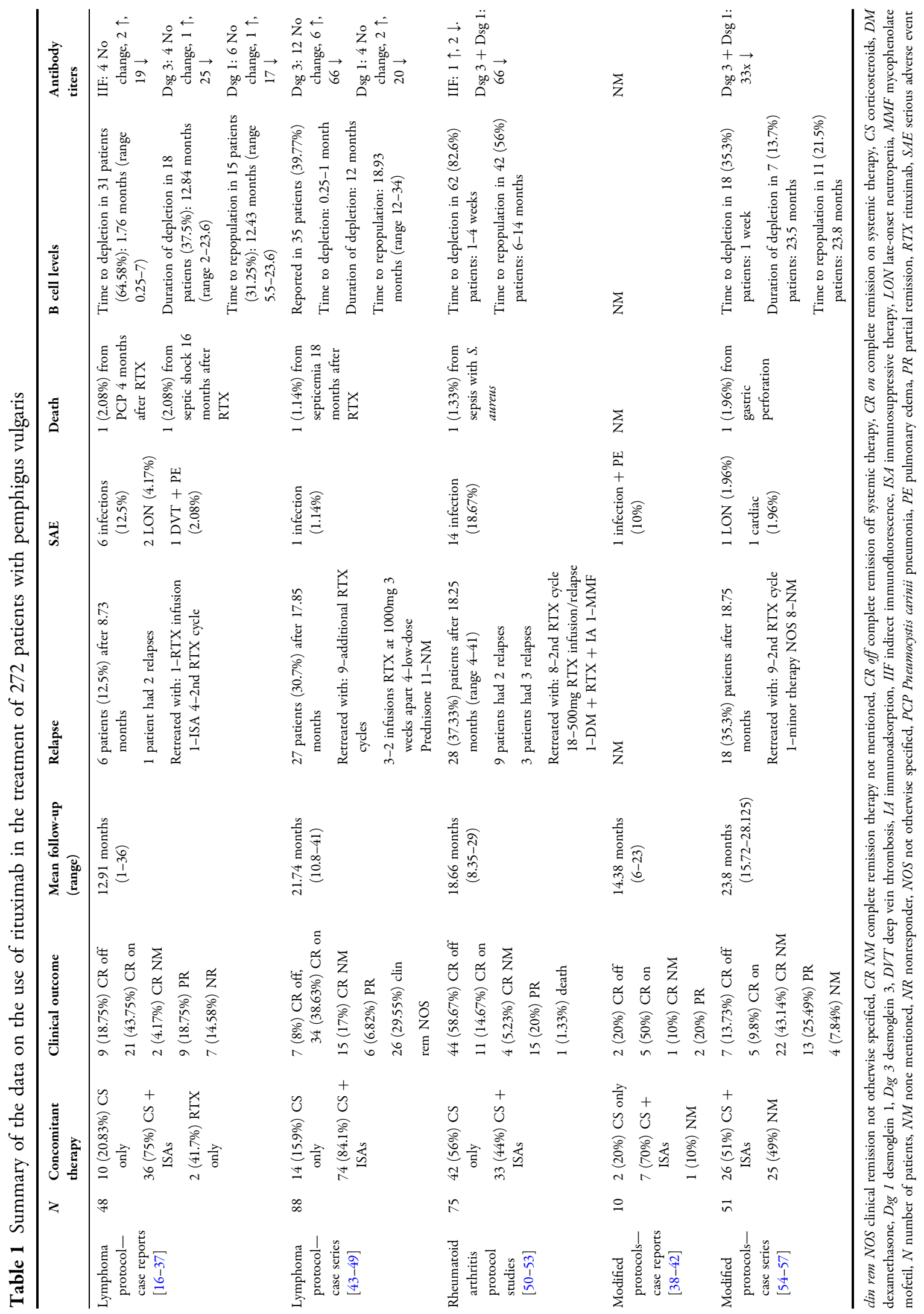


Six (12.5\%) patients had seven relapses after a mean time of 8.73 months (range 1.5-12 months) after discontinuing rituximab $[16,22,30,31,33]$. One patient relapsed twice and received an infusion of rituximab each time. One patient received ISAs, while the remaining four patients received a second cycle of rituximab.

Serious adverse events reported included one death from Pneumocystis carinii pneumonia 4 months after rituximab [21], one death from septic shock after 16 months [36], one sepsis with multidrug-resistant Pseudomonas aeruginosa, Enterococcus faecalis, and Staphylococcus aureus [16], one bacterial pneumonia [22], one recurrence of $P$. aeruginosa hip arthritis [22], one severe late-onset neutropenia after 27 weeks [28], one late-onset neutropenia and bacterial pneumonia after 19 weeks [29], one cytomegalovirus gastritis and retinitis [29], one deep vein thrombosis and pulmonary embolism [33], and one $P$. carinii pneumonia [33].

The time to depletion of B cells (undetectable levels in peripheral blood) after the first rituximab infusion was available for 31 (64.58\%) patients and varied from 1 week to 7 months (mean 1.76 months) [16, 17, 20, 22-24, 26, 27, 29-31, 33, 35]. The mean duration of depletion of $\mathrm{B}$ cells was available for $18(37.5 \%)$ patients and was 12.84 months (range 2-23.6 months) [16, 17, 20, 22, 24, 26, $27,29,33]$. The mean time for repopulation of $B$ cells (return to levels present in the peripheral blood before rituximab therapy) was available for 15 (31.25\%) patients and was 12.43 months (range 5.5-23.6 months) [20, 22, 24, 26, 30, 31, 33].

Of the $15(31.25 \%)$ patients reported with indirect immunofluorescence (IIF) only, two patients' titers remained unchanged throughout the study period, one of whom had two relapses $[16,17]$. Two patients had an increase in their titers, one of whom relapsed as the titers increased while the other relapsed 5 months earlier than the increase [22]. Eleven patients had a decrease in titers at the end of the study period with no relapses reported [21-23, $29,34]$. In the $20(41.67 \%)$ patients in whom enzyme-linked immunosorbent assay (ELISA) for Dsg 1 and Dsg 3 were performed, decreases in titers were observed with rituximab therapy and clinical response [26, 30, 31, 33, 35, 37]. A similar pattern was observed in 10 (20.83\%) patients in whom both IIF and ELISA data were available [18, 20, 24, 25, 27, 32].

\section{Case Series}

Data on 88 patients were reported in seven case series [43-49]. Clinical response was as follows: complete response was observed in 56 (63.63\%) patients; seven (8\%) patients were off therapies; 34 (38.63\%) patients were on therapy; and 15 (17\%) patients had an unclear therapy status. Six $(6.82 \%)$ patients had partial remission. Twenty-six (29.55\%) patients improved but the definition of improvement was undefined. Nonresponders were not reported. The mean follow-up was 21.75 months (range 10.8-41 months).

Twenty-seven (30.68\%) patients relapsed 29 times after a mean of 17.85 months (range 6-34 months) after discontinuation of rituximab [43, 45-49]. Nine of these patients were re-treated with additional rituximab cycles. Three patients were treated with two rituximab infusions $1,000 \mathrm{mg}$ each, 3 weeks apart. Four patients received low-dose prednisone.

Serious adverse events included one death from septicemia after 18 months [43], and one pyelonephritis 12 months after discontinuation of rituximab [43].

Depletion of B cells was reported in 35 patients and occurred between 1 and 4 weeks, 
and lasted up to 12 months [43-45]. Time for B cell repopulation occurred between 12 and 34 months (mean 18.93 months). The data suggest that the probability of relapse is higher in patients who take longer to repopulate.

In the majority of patients, a decrease in Dsg 3 titers was reported [43-49]. Nonetheless, 11 (12.5\%) patients had persistently high titers while in clinical remission [43, 49]. Also, six patients who experienced a relapse at 12 and 18 months had increased titers at the time of relapse [48]. In 28 (31.8\%) patients rituximab therapy resulted in a decrease in Dsg 1 antibody titers [43, 44, 47].

\section{The Rheumatoid Arthritis Protocol}

\section{Case Series}

Data on 75 patients were reported in four studies [50-53]. Complete remission was reported in 59 (78.67\%) patients, of whom 44 $(58.67 \%)$ were off therapy, $11(14.67 \%)$ on therapy, and in four patients (5.33\%) the therapy was unclear. Fifteen (20\%) patients had partial remission, and one (1.33\%) patient died. The mean duration of follow-up was 18.66 months (range 8.35-29 months).

Twenty-eight (37.33\%) patients had 43 relapses [51-53]. Nine patients had two relapses. Three patients relapsed three times [53]. Relapses were treated with rituximab with success. In some patients, corticosteroids, ISAs, and immunoadsorption were used.

Adverse events occurred in 15 patients (20\%) [50-53]. These included one death from sepsis with $S$. aureus [50]. Two other patients had sepsis [50, 51], one of whom had spinal hemorrhage with transient paraplegia of both legs, three had pneumonias [52, 53], six urinary tract infections [53], one extensive herpes simplex infection [51], one herpes keratitis [53], and one herpes zoster [53].
B cell levels were depleted within 1-4 weeks [51-53]. Approximately $80 \%$ of these were complete responders and $20 \%$ were partial responders.

IIF decreased in two patients studied [52]. In one patient, a relapse was accompanied by a rise in the titer [52]. ELISA levels decreased in some patients [50, 51, 53].

\section{Modified Protocols}

\section{Case Reports}

In ten patients, the modified lymphoma protocol was used [38-42]. Patients receiving modified lymphoma protocols received three or four additional monthly infusions and one received a complete second cycle of the protocol. Eight (80\%) patients had complete remission, two (20\%) had partial remission. None of the patients had relapses. Patients received corticosteroids or corticosteroids and ISAs as concomitant therapies. One patient experienced a bacterial pneumonia and pulmonary embolism [39].

B cell studies were not reported and antibody titers determined by IIF and ELISA decreased [38, 39, 41].

One study reported three patients treated with rituximab without details of the protocol [58]. They were not included in the analysis.

\section{Case Series}

Data on 51 patients were presented in four studies [54-57]. In the first study, six patients received eight weekly infusions of $375 \mathrm{mg} / \mathrm{m}^{2}$ followed by a single dose once a month for 4 months [55]. All six had a complete remission off therapy. In 25 patients, two separate protocols were used [56]. One group received $375 \mathrm{mg} / \mathrm{m}^{2}$ in two infusions in 2 weeks. Half of the patients had a complete remission and the other half had a partial remission. In the second 
group, patients got three or more weekly infusions of $375 \mathrm{mg} / \mathrm{m}^{2}$, and $90 \%$ had complete remission while $10 \%$ had partial remission. In the third study, 12 patients received the RA protocol at a dose of $500 \mathrm{mg}$ at 2 week intervals [54]. Six of the 12 had complete remission off and on therapy, and six had partial remission. The fourth study concerned eight patients [57]. Of the four patients who got the RA protocol at a dose of $1,000 \mathrm{mg}$ at 2 weeks, two had complete remission and two had partial remission. One patient received two infusions of $500 \mathrm{mg}$ at 2 week intervals and had a complete remission. The remaining three patients received the lymphoma protocol, two had complete remission and one had a partial remission.

Among the 51 patients in the modified protocol group, 18 (35.3\%) patients had relapses after a mean of 18.75 months (range 11.5-24.25 months) [54, 56, 57]. Half were treated with a second cycle of rituximab and experienced a complete remission [54, 57]. Serious adverse events were reported in three $(5.8 \%)$ patients $[54,56]$. One had gastric perforation resulting in death [56], one had cardiac complications [54], and one had sepsis with neutropenia 7.25 months later [54].

Depletion of B cells lasted up to 40 months in studies that reported it $[54,55]$. In seven patients, repopulation was observed after 20-35 months. In five (71\%) of these patients, repopulation was accompanied by relapses.

None of the studies reported IIF. ELISA for both Dsg 1 and Dsg 3 reported decreases [56, 57].

\section{DISCUSSION}

The analysis of the available literature on the use of rituximab in treating patients with PV is not only difficult but restrictive, as a direct consequence of the significant limitations of the data. Therefore, interpretation of the data analysis must be done in the light and perspective of these limitations. An obvious inference would be that such an analysis could be of limited value. On the contrary, these limitations are of significant benefit, because they will help in the design of future studies, and focus on elements of the pharmacodynamics of rituximab therapy.

Some of these limitations are as follows: the data come from multiple sources; there is a significant lack of uniformity in the selection of the patients, in defining their severity or extent of disease, and in identifying failed treatment before rituximab; the lack of an objective scoring system makes changes or responses difficult to evaluate numerically; the more concerning aspect is the limited follow-up provided by most authors, this becomes an important issue because significant side effects and relapses can occur several months after the discontinuation of rituximab therapy.

As rituximab is a B cell depletion therapy, many authors have not provided any data on $\mathrm{B}$ cell levels and the changes in autoantibody levels. None of the studies provide any rationale or scientific basis for the use of the lymphoma or RA protocols in treating patients with PV.

The response of patients to rituximab using the lymphoma protocol or RA protocol has been described in the results section. The total number of patients treated by the lymphoma protocol and its modification was 180 . Ninetytwo patients were treated with the RA protocol including its modifications. The length of followup for patients in the lymphoma protocol was a mean of 15.44 months (range 1-41 months) and 21.04 months (range 8.35-29 months) for the RA protocol. These figures permit preliminary conclusions. A complete remission occurred in 
$66.66 \%$ of patients on the lymphoma protocol and $75 \%$ in the RA protocol. This would suggest that both protocols are effective in producing clinical remissions during an 18-month followup. Using the lymphoma protocol during this period, $11.11 \%$ of these patients are off therapy and $33.33 \%$ are on therapy. In contrast, in the RA protocol, $53.26 \%$ are off therapy and $17.4 \%$ are on therapy. This apparent difference is partly due to the fact that the number of patients in whom the presence or absence of therapy while in complete remission is not mentioned in $22.22 \%$ of the patients in the lymphoma protocol, but only in $4.34 \%$ of the patients in the RA protocol. Interestingly, a partial response was observed in $12.78 \%$ of patients in the lymphoma protocol, but $23.91 \%$ of the patients in the RA protocol. Of significant interest is the fact that there were no nonresponders in patients treated with the RA protocol compared to $3.9 \%$ in the lymphoma protocol. The relapse rates were $22.78 \%$ in the lymphoma protocol and $35.87 \%$ in the RA protocol. The incidence of serious infections was $3.9 \%$ in the lymphoma protocol but $15.21 \%$ in the RA protocol. The mortality rate in the lymphoma protocol was $2.22 \%$ and $1.09 \%$ in the RA protocol. The difference between the two protocols in these important variables is striking and noteworthy. It is thus clear that there are significant and remarkable differences in the patient responses between the two protocols. The data analysis did not provide clear indications for specific reasons that may account for these differences.

Preliminary observations would suggest that while the use of the lymphoma protocol produces a lower response rate, there is a lower rate of recurrences and serious infections but a higher mortality rate. Patients treated by the RA protocol had higher response rates, a larger number of infections, but a lower mortality rate. This could be partly due to the fact that more patients were on corticosteroids and immunosuppressive agents as concomitant therapy in the lymphoma protocol, thus adding to the degree and duration of prolonged immune suppression.

To serve the best interests of the patient, it is useful and relevant to determine what purpose the lymphoma and RA protocols serve in their respective diseases. The use of rituximab in lymphoma patients has a duration of response of a median of 12 months (range 11-13.4 months) after which relapses frequently occur [59]. A median progression-free survival of 18 months and a 5-year relapse free survival of $28 \%$ was reported [59]. The most relevant use of rituximab comes from a 3-year progression-free survival study in which patients were given cyclophosphamide, doxorubicin, vincristine, and prednisone-like chemotherapy with or without rituximab and the 3-year survival rates were $93 \%$ and $84 \%$, respectively [60]. Therefore, to obtain the benefit of rituximab in lymphoma patients, the addition of other chemotherapeutic agents is required, and can be best measured in 3-year survival rates.

The use of rituximab in RA is still a matter of debate and discussion. Between 2006 and 2011, 5,903 patients, who were reported in eight different trials, were treated [61-68]. These studies compared various parameters. The benefits of rituximab are measured by the American College of Rheumatology's 20\%, 50\% and 70\% improvement criteria [61-68]. The only statistically significant dose regime was two cycles of $1,000 \mathrm{mg}$ of rituximab over 48 weeks that achieved an American College of Rheumatology improvement of $20 \%$. The Rituximab Consensus Expert Committee in 2011 stated that the optimal treatment paradigms have not yet been defined [69]. Recently, two extremely different treatment options have been suggested. In the treatment to target protocol, rituximab is given to keep the 
disease activity score at 2.6 or less whether clinically needed or not. In the other protocol, rituximab is given on an as-needed basis [70]. Some patients need five cycles or more to maintain clinical remission [71-73]. However, at the present time, there is no protocol that provides prolonged and sustained remission in RA. Therefore, it is unclear how many cycles are required to keep patients symptom free, prevent joint destruction, or can be safely given in any defined period. Furthermore, RA patients usually receive rituximab with methotrexate concomitantly and often additional prednisone [61-68]. PV treated by the RA protocol needed multiple infusions to treat recurrent relapses [52, 53].

The objectives of treating patients with PV are different from those with lymphoma or RA. The optimal treatment for PV is one in which the disease is controlled, relapses are prevented, and the long-term sustained clinical remission without continued treatment can be achieved.

In patients with PV and ocular cicatricial pemphigoid, a defined protocol with a definitive endpoint was used $[74,75]$. This combination of rituximab and intravenous immunoglobulin allowed for the discontinuation of previous systemic corticosteroids and ISAs and produced sustained clinical remissions. There were no infections or deaths.

There is a growing trend among dermatologists to use rituximab. Many variables have yet to be determined or defined. Providing guidelines or indications for therapy is preliminary and may change in the future. Presently, the indications for using rituximab could be as follows.

- Failure of conventional therapy for minimally 6 months.

- When conventional therapy has failed, or produced significant and catastrophic side effects, or is contraindicated, then intravenous immunoglobulin may be used [76].

- Active or latent infections are a definitive contraindication.

- Before initiating rituximab therapy, the goals and the endpoint should be discussed. The long-term side effects of rituximab in patients with PV are not yet known. Rituximab can result in cardiac side effects $[77,78]$. Patients and their families should be advised of these facts.

- As clinical response may be faster with rituximab compared to conventional therapy, patients should be advised to return at frequent intervals for the early detection of possible recurrences and also for monitoring of late-onset side effects.

\section{CONCLUSION}

The use of rituximab in PV is an evolving work in progress. Although the data have limitations, the drug is effective in controlling recalcitrant disease. Whether this control is long term, life long, or of limited duration is not yet known. The data suggest that following either the lymphoma or RA protocols is not optimal or particularly advantageous, and modifications of both are warranted. Both carry a risk of severe and possibly fatal infections. Presently, it should be the treatment of last resort. Monitoring the levels of B cells (CD19+/CD20+) in the peripheral blood and immunoglobulin levels during and after therapy is advisable. A protocol that is unique for autoimmune mucocutaneous blistering disease is needed. Preliminary studies using such a protocol show promising results $[74,75]$. Rituximab is the new frontier for the treatment of PV. 


\section{ACKNOWLEDGMENTS}

Dr. Ahmed is the guarantor for this article, and takes responsibility for the integrity of the work as a whole.

Conflict of interest. The authors have no conflicts of interest to report.

Open Access. This article is distributed under the terms of the Creative Commons Attribution Noncommercial License which permits any noncommercial use, distribution, and reproduction in any medium, provided the original author(s) and the source are credited.

\section{REFERENCES}

1. Nousari HC, Anhalt GJ. Pemphigus and bullous pemphigoid. Lancet. 1999;354:667-72.

2. Yeh SW, Sami N, Ahmed RA. Treatment of pemphigus vulgaris: current and emerging options. Am J Clin Dermatol. 2005;6:327-42.

3. Sami N. Approach to the patient with autoimmune mucocutaneous blistering diseases. Dermatol Ther. 2011;24:173-86.

4. Kneisel A, Hertl M. Autoimmune bullous skin diseases. Part 1: clinical manifestations. J Dtsch Dermatol Ges. 2011;9:844-56; quiz 57.

5. Groves RW. Pemphigus: a brief review. Clin Med. 2009;9:371-5.

6. Korman NJ. Pemphigus. Dermatol Clin. 1990;8:689-700.

7. Sami N, Ahmed AR. Dual diagnosis of pemphigus and pemphigoid. Retrospective review of thirty cases in the literature. Dermatology. 2001;202:293-301.

8. Scott JE, Ahmed AR. The blistering diseases. Med Clin North Am. 1998;82:1239-83.

9. Darling MR, Daley T. Blistering mucocutaneous diseases of the oral mucosa-a review: part 2 . Pemphigus vulgaris. J Can Dent Assoc. 2006;72:63-6.
10. Kasperkiewicz M, Schmidt E. Current treatment of autoimmune blistering diseases. Curr Drug Discov Technol. 2009;6:270-80.

11. Toth GG, Jonkman MF. Therapy of pemphigus. Clin Dermatol. 2001;19:761-7.

12. Feldman RJ, Ahmed AR. Relevance of rituximab therapy in pemphigus vulgaris: analysis of current data and the immunologic basis for its observed responses. Expert Rev Clin Immunol. 2011;7:529-41.

13. Food and Drug Administration. Rituxan Label. 2012. http://www.accessdata.fda.gov/drugsatfda docs/label/2012/103705s5373lbl.pdf. Accessed Oct 152012 .

14. Gurcan HM, Keskin DB, Stern JN, et al. A review of the current use of rituximab in autoimmune diseases. Int Immunopharmacol. 2009;9:10-25.

15. Murrell DF, Dick S, Ahmed AR, et al. Consensus statement on definitions of disease, end points, and therapeutic response for pemphigus. J Am Acad Dermatol. 2008;58:1043-6.

16. Salopek TG, Logsetty S, Tredget EE. Anti-CD20 chimeric monoclonal antibody (rituximab) for the treatment of recalcitrant, life-threatening pemphigus vulgaris with implications in the pathogenesis of the disorder. J Am Acad Dermatol. 2002;47:785-8.

17. Cooper HL, Healy E, Theaker JM, Friedmann PS. Treatment of resistant pemphigus vulgaris with an anti-CD20 monoclonal antibody (Rituximab). Clin Exp Dermatol. 2003;28:366-8.

18. Herrmann G, Hunzelmann N, Engert A. Treatment of pemphigus vulgaris with anti-CD20 monoclonal antibody (rituximab). $\mathrm{Br} \mathrm{J}$ Dermatol. 2003;148:602-3.

19. Virgolini L, Marzocchi V. Anti-CD20 monoclonal antibody (rituximab) in the treatment of autoimmune diseases. Successful result in refractory pemphigus vulgaris: report of a case. Haematologica. 2003;88:ELT24.

20. Espana A, Fernandez-Galar M, Lloret P, SanchezIbarrola A, Panizo C. Long-term complete remission of severe pemphigus vulgaris with monoclonal antiCD20 antibody therapy and immunophenotype correlations. J Am Acad Dermatol. 2004;50:974-6.

21. Morrison LH. Therapy of refractory pemphigus vulgaris with monoclonal anti-CD20 antibody (rituximab). J Am Acad Dermatol. 2004;51:817-9.

22. Dupuy A, Viguier M, Bedane C, et al. Treatment of refractory pemphigus vulgaris with rituximab (anti- 
CD20 monoclonal antibody). Arch Dermatol. 2004;140:91-6.

23. Wenzel J, Bauer R, Bieber T, Tuting T. Successful rituximab treatment of severe pemphigus vulgaris resistant to multiple immunosuppressants. Acta Derm Venereol. 2005;85:185-6.

24. Arin MJ, Engert A, Krieg T, Hunzelmann N. AntiCD20 monoclonal antibody (rituximab) in the treatment of pemphigus. $\mathrm{Br} \mathrm{J}$ Dermatol. 2005; 153:620-5.

25. Cecchi R, Gasperini U. Severe pemphigus vulgaris treated with rituximab (Mabthera). J Dermatol. $2005 ; 32: 862-4$.

26. Niedermeier A, Worl P, Barth S, Schuler G, Hertl M. Delayed response of oral pemphigus vulgaris to rituximab treatment. Eur J Dermatol. 2006;16:266-70.

27. Esposito M, Capriotti E, Giunta A, Bianchi L, Chimenti S. Long-lasting remission of pemphigus vulgaris treated with rituximab. Acta Derm Venereol. 2006;86:87-9.

28. Rios-Fernandez R, Gutierrez-Salmeron MT, CallejasRubio JL, Fernandez-Pugnaire M, Ortego-Centeno $\mathrm{N}$. Late-onset neutropenia following rituximab treatment in patients with autoimmune diseases. Br J Dermatol. 2007;157:1271-3.

29. Goh MS, McCormack C, Dinh HV, et al. Rituximab in the adjuvant treatment of pemphigus vulgaris: a prospective open-label pilot study in five patients. Br J Dermatol. 2007;156:990-6.

30. Antonucci A, Negosanti M, Tabanelli M, Varotti C. Treatment of refractory pemphigus vulgaris with anti-CD20 monoclonal antibody (rituximab): five cases. J Dermatolog Treat. 2007;18:178-83.

31. Marzano AV, Fanoni D, Venegoni L, Berti E, Caputo $\mathrm{R}$. Treatment of refractory pemphigus with the antiCD20 monoclonal antibody (rituximab). Dermatology. 2007;214:310-8.

32. Weger $W$, Aberer E. Treatment failure with rituximab in a patient with pemphigus vulgaris. J Eur Acad Dermatol Venereol. 2008;22:387-9.

33. Shimanovich I, Nitschke M, Rose C, Grabbe J, Zillikens D. Treatment of severe pemphigus with protein A immunoadsorption, rituximab and intravenous immunoglobulins. $\mathrm{Br} \mathrm{J}$ Dermatol. 2008;158:382-8.

34. Wollina U, Koch A, Hansel G. Rituximab therapy of recalcitrant bullous dermatoses. J Dermatol Case Rep. 2008;2:4-7.
35. Sorce M, Arico M, Bongiorno MR. Rituximab in refractory pemphigus vulgaris. Dermatol Ther. 2008;21:S6-9.

36. Tournadre A, Amarger S, Joly P, et al. Polymyositis and pemphigus vulgaris in a patient: successful treatment with rituximab. Jt Bone Spine. 2008;75:728-9.

37. Pfutze M, Eming R, Kneisel A, et al. Clinical and immunological follow-up of pemphigus patients on adjuvant treatment with immunoadsorption or rituximab. Dermatology. 2009;218:237-45.

38. Belgi AS, Azeez M, Hoyle C, Williams RE. Response of pemphigus vulgaris to anti-CD20 antibody therapy (rituximab) may be delayed. Clin Exp Dermatol. 2006;31:143.

39. Schmidt E, Seitz CS, Benoit S, Brocker EB, Goebeler M. Rituximab in autoimmune bullous diseases: mixed responses and adverse effects. $\mathrm{Br} \mathrm{J}$ Dermatol. 2007;156:352-6.

40. Barrera MV, Mendiola MV, Bosch RJ, Herrera E. Prolonged treatment with rituximab in patients with refractory pemphigus vulgaris. J Dermatolog Treat. 2007;18:312-4.

41. Faurschou A, Gniadecki R. Two courses of rituximab (anti-CD20 monoclonal antibody) for recalcitrant pemphigus vulgaris. Int J Dermatol. 2008;47:292-4.

42. Craythorne EE, Mufti G, DuVivier AW. Rituximab used as a first-line single agent in the treatment of pemphigus vulgaris. J Am Acad Dermatol. 2011;65:1064-5.

43. Joly P, Mouquet $\mathrm{H}$, Roujeau JC, et al. A single cycle of rituximab for the treatment of severe pemphigus. N Engl J Med. 2007;357:545-52.

44. Cianchini G, Corona R, Frezzolini A, et al. Treatment of severe pemphigus with rituximab: report of 12 cases and a review of the literature. Arch Dermatol. 2007;143:1033-8.

45. Eming R, Nagel A, Wolff-Franke S, et al. Rituximab exerts a dual effect in pemphigus vulgaris. J Invest Dermatol. 2008;128:2850-8.

46. Nagel A, Podstawa E, Eickmann M, et al. Rituximab mediates a strong elevation of B-cell-activating factor associated with increased pathogen-specific IgG but not autoantibodies in pemphigus vulgaris. J Invest Dermatol. 2009;129:2202-10.

47. Schmidt E, Hennig $\mathrm{K}$, Mengede C, Zillikens D, Kromminga A. Immunogenicity of rituximab in patients with severe pemphigus. Clin Immunol. 2009;132:334-41. 
48. Muller R, Hunzelmann N, Baur V, et al. Targeted immunotherapy with rituximab leads to a transient alteration of the IgG autoantibody profile in pemphigus vulgaris. Dermatol Res Pract. 2010 (Epub ahead of print).

49. Reguiai Z, Tabary T, Maizieres M, Bernard P. Rituximab treatment of severe pemphigus: longterm results including immunologic follow-up. J Am Acad Dermatol. 2012;67:623-9.

50. Kanwar AJ, Tsuruta D, Vinay K, et al. Efficacy and safety of rituximab treatment in Indian pemphigus patients. J Eur Acad Dermatol Venereol. 2011 (Epub ahead of print).

51. Kasperkiewicz M, Shimanovich I, Meier M, et al. Treatment of severe pemphigus with a combination of immunoadsorption, rituximab, pulsed dexamethasone and azathioprine/mycophenolate mofetil: a pilot study of 23 patients. $\mathrm{Br} \mathrm{J}$ Dermatol. 2012;166:154-60.

52. Matsukura S, Knowles SR, Walsh S, Shear NH. Effect of a single-cycle alternative dosing regimen for rituximab for recalcitrant pemphigus: a case series of 9 patients. Arch Dermatol. 2012;148:734-9.

53. Cianchini G, Lupi F, Masini C, et al. Therapy with rituximab for autoimmune pemphigus: results from a single-center observational study on 42 cases with long-term follow-up. J Am Acad Dermatol. 2012;67:617-22.

54. Horvath B, Huizinga J, Pas HH, Mulder AB, Jonkman MF. Low-dose rituximab is effective in pemphigus. Br J Dermatol. 2012;166:405-12.

55. Craythorne E, du Viver A, Mufti GJ, Warnakulasuriya S. Rituximab for the treatment of corticosteroid-refractory pemphigus vulgaris with oral and skin manifestations. J Oral Pathol Med. 2011;40:616-20.

56. Kim JH, Kim YH, Kim MR, Kim SC. Clinical efficacy of different doses of rituximab in the treatment of pemphigus: a retrospective study of 27 patients. Br J Dermatol. 2011;165:646-51.

57. Kasperkiewicz M, Shimanovich I, Ludwig RJ, et al. Rituximab for treatment-refractory pemphigus and pemphigoid: a case series of 17 patients. J Am Acad Dermatol. 2011;65:552-8.

58. Strowd LC, Taylor SL, Jorizzo JL, Namazi MR. Therapeutic ladder for pemphigus vulgaris: emphasis on achieving complete remission. J Am Acad Dermatol. 2011;64:490-4.

59. Molina A. A decade of rituximab: improving survival outcomes in non-Hodgkin's lymphoma. Annu Rev Med. 2008;59:237-50.
60. Maloney DG. Anti-CD20 antibody therapy for B-cell lymphomas. N Engl J Med. 2012;366:2008-16.

61. Cohen SB, Emery P, Greenwald $\mathrm{MW}$, et al. Rituximab for rheumatoid arthritis refractory to anti-tumor necrosis factor therapy: results of a multicenter, randomized, double-blind, placebocontrolled, phase III trial evaluating primary efficacy and safety at twenty-four weeks. Arthritis Rheum. 2006;54:2793-806.

62. Edwards JC, Szczepanski L, Szechinski J, et al. Efficacy of B-cell-targeted therapy with rituximab in patients with rheumatoid arthritis. $\mathrm{N}$ Engl J Med. 2004;350:2572-81.

63. Emery P, Deodhar A, Rigby WF, et al. Efficacy and safety of different doses and retreatment of rituximab: a randomised, placebo-controlled trial in patients who are biological naive with active rheumatoid arthritis and an inadequate response to methotrexate (Study Evaluating Rituximab's Efficacy in MTX iNadequate rEsponders (SERENE)). Ann Rheum Dis. 2011;69:1629-35.

64. Emery P, Fleischmann R, Filipowicz-Sosnowska A, et al. The efficacy and safety of rituximab in patients with active rheumatoid arthritis despite methotrexate treatment: results of a phase IIB randomized, double-blind, placebo-controlled, dose-ranging trial. Arthritis Rheum. 2006;54:1390-400.

65. Haraoui B, Bokarewa M, Kallmeyer I, Bykerk VP. Safety and effectiveness of rituximab in patients with rheumatoid arthritis following an inadequate response to 1 prior tumor necrosis factor inhibitor: the RESET Trial. J Rheumatol. 2011;38:2548-56.

66. Mease PJ, Cohen S, Gaylis NB, et al. Efficacy and safety of retreatment in patients with rheumatoid arthritis with previous inadequate response to tumor necrosis factor inhibitors: results from the SUNRISE trial. J Rheumatol. 2010;37:917-27.

67. Rubbert-Roth A, Tak PP, Zerbini C, et al. Comment on: efficacy and safety of various repeat treatment dosing regimens of rituximab in patients with active rheumatoid arthritis: results of a phase III randomized study (MIRROR). Rheumatology. 2010;49:1683-93.

68. Tak PP, Rigby W, Rubbert-Roth A, et al. Sustained inhibition of progressive joint damage with rituximab plus methotrexate in early active rheumatoid arthritis: 2-year results from the randomised controlled trial IMAGE. Ann Rheum Dis. 2012;71:351-7.

69. Buch MH, Smolen JS, Betteridge N, et al. Updated consensus statement on the use of rituximab in 
patients with rheumatoid arthritis. Ann Rheum Dis. 2011;70:909-20.

70. Emery P, Mease PJ, Rubbert-Roth A, et al. Retreatment with rituximab based on a treatmentto-target approach provides better disease control than treatment as needed in patients with rheumatoid arthritis: a retrospective pooled analysis. Rheumatology. 2011;50:2223-32.

71. Keystone E, Fleischmann R, Emery P, et al. Safety and efficacy of additional courses of rituximab in patients with active rheumatoid arthritis: an openlabel extension analysis. Arthritis Rheum. 2007;56:3896-908.

72. Popa C, Leandro MJ, Cambridge G, Edwards JC. Repeated B lymphocyte depletion with rituximab in rheumatoid arthritis over 7 years. Rheumatology. 2007;46:626-30.

73. van Vollenhoven RF, Emery P, Bingham CO III, et al. Longterm safety of patients receiving rituximab in rheumatoid arthritis clinical trials. J Rheumatol. 2010;37:558-67.
74. Ahmed AR, Spigelman Z, Cavacini LA, Posner MR. Treatment of pemphigus vulgaris with rituximab and intravenous immune globulin. N Engl J Med. 2006;355:1772-9.

75. Foster CS, Chang PY, Ahmed AR. Combination of rituximab and intravenous immunoglobulin for recalcitrant ocular cicatricial pemphigoid: a preliminary report. Ophthalmology. 2010;117:861-9.

76. Ahmed AR, Dhal MV. Consensus statement on the use of intravenous immunoglobulin therapy in the treatment of autoimmune mucocutaneous blistering diseases. Arch Dermatol. 2003;139:1051-9.

77. Lourari S, Herve C, Doffoel-Hantz V, et al. Bullous and mucous membrane pemphigoid show a mixed response to rituximab: experience in seven patients. J Eur Acad Dermatol Venereol. 2011;25:1238-40.

78. Garypidou V, Perifanis V, Tziomalos K, Theodoridou S. Cardiac toxicity during rituximab administration. Leuk Lymphoma. 2004;45:203-4. 